\title{
Impacts des facteurs climatiques et anthropiques sur les ressources et la qualité des eaux de la mare de Tabalak
}

\author{
Mahaman Moustapha ADAMOU ${ }^{1 *}$, Bassirou A LHOU ${ }^{2}$, \\ Yahaya NAZOUMOU ${ }^{3}$ et Gilbert ALLOKE ${ }^{1}$ \\ ${ }^{1}$ Faculté d'Agronomie, Université Abdou Moumouni de Niamey, BP 10960, Niamey, Niger. \\ ${ }^{2}$ Ecole Normale Supérieure, Département Sciences de la Vie et de la Terre. \\ ${ }^{3}$ Faculté des Sciences et Techniques, Université Abdou Moumouni de Niamey, BP 10960, Niamey, Niger. \\ *Auteur correspondant, E-mail : moustapha_a@yahoo.com
}

\section{RESUME}

Au Niger, les facteurs climatiques et anthropiques influencent les secteurs de l'agriculture, de l'élevage et de la pêche et la qualité des eaux de surface. Cette étude a pour objectif d'identifier les impacts des aléas climatiques et de l'action de l'homme sur les ressources et la qualité de l'eau de la mare de Tabalak. A terme, cette étude devrait permettre de poser les jalons pour des actions, afin de réduire les risques liés à l'exploitation des ressources de cette zone humide. Pour réaliser cette étude, des enquêtes, des visites de terrain et des prélèvements d'échantillons d'eau ont été effectuées. Ces derniers ont été analysés au laboratoire. L'analyse des données météorologiques et des perceptions de la population sur la variabilité climatique indiquent une tendance à la baisse des précipitations contre une hausse des températures et de la force des vents. En ce qui concerne la dynamique d'occupation des sols, toutes les unités ont subi des transformations. Selon $80 \%$ des personnes enquêtées, cette tendance est causée par des facteurs climatiques et anthropiques. Les impacts de ces aléas sont : une perturbation dans le déroulement des saisons, une baisse de la productivité, une perte des terres par ravinement ; la formation de dunes vives; l'ensablement et l'assèchement fréquent de la mare, la pression parasitaire et la persistance des épizooties. Les résultats sur la qualité des eaux montrent que les paramètres physico-chimiques étudiés se situent dans les normes requises. Cependant, la présence des métaux lourds tels que le fer, le zinc, le nickel, le plomb, le manganèse et le chrome, dénote de l'existence de sources de pollutions diffuses. Au terme de cette étude, le constat général montre que l'environnement biophysique du bassin versant de la mare de Tabalak présente de sérieuses tendances à la dégradation des terres. Ces tendances dont les conséquences directes affectent le potentiel productif du bassin versant de la mare sont particulièrement dues aux facteurs climatiques et à la surexploitation des ressources.

(C) 2015 International Formulae Group. All rights reserved.

Mots clés : Climat, agriculture, élevage, pêche, qualité des eaux, Tabalak.

\section{INTRODUCTION}

Pays sahélien, le Niger enregistre depuis le début des années soixante dix une baisse de pluviométrie sur plusieurs stations météorologiques, alors que les températures sont en augmentation (PNUD/BCPR, 2013).
Malgré cette situation, le pays dispose de nombreuses ressources naturelles, notamment les zones humides reparties sur tout le territoire, y compris dans la région saharienne au Nord. Ces zones humides renferment des systèmes agro-sylvo-pastoraux importants qui 
jouent un rôle vital dans la lutte contre la pauvreté, l'insécurité alimentaire, la préservation de la biodiversité et le maintien de l'équilibre des écosystèmes. En plus, elles abritent une grande diversité de plantes et d'animaux, en particulier les oiseaux d'eau (Alhouza, 2010).

Cependant, au cours des trente dernières années, plusieurs zones humides sont en voie de disparition. L'insuffisance de la pluviométrie, les assèchements, les drainages et les mises en culture sont les principales causes qui mettent en danger l'équilibre hydrologique et biologique de ces écosystèmes (Ramsar, 2013). En outre, les zones humides sont l'un des secteurs les plus vulnérables à la variabilité climatique et aux actions anthropiques, ce qui influence directement les secteurs tels que l'agriculture, l'élevage et la pêche (PNUD/BCPR, 2013 ; CNEDD, 2011).

C'est conscient de tout cela que, depuis plus d'une décennie, le Niger a inscrit les questions de gestion de ressources naturelles notamment les zones humides au centre des politiques et des stratégies nationales de développement. C'est ainsi qu'il a ratifié en 1987 la convention Ramsar sur les zones humides d'importance internationale. Par cet acte, le Niger s'engage à utiliser de façon rationnelle les ressources de ces écosystèmes particuliers par des modes de gestion durable permettant de concilier les activités sociales et économiques avec le maintien de l'équilibre naturel. A ce jour, douze (12) zones humides du Niger ont été inscrites sur la liste Ramsar et considérées comme sites d'importance internationale ou «sites Ramsar», dont la mare de Tabalak en septembre 2005 (MHE/LCD, 2006). Cependant, cette zone humide est en proie à diverses menaces d'ordre anthropiques et climatiques qui à terme peuvent anéantir ses multiples fonctions et services.

C'est pour mieux cerner l'action du climat et de l'homme sur la mare que cette étude a été entreprise. Elle permettra de bien cerner les aléas climatiques actuels de la zone, afin de mieux proposer des options d'adaptation en vue de diminuer leurs impacts sur les différents secteurs. Elle permettra aussi de poser les premiers jalons pour un meilleur suivi de la qualité des eaux de la mare de Tabalak.

\section{MATERIEL ET METHODES \\ Zone d'étude}

La mare de Tabalak est localisée dans la région de Tahoua et est à cheval entre les communes de Tabalak (département d'Abalak), Kalfou (département de Tahoua) et Keita (département de Keita) (Figure1).

Le bassin versant de la mare couvre une superficie de 109141 ha et fait partie de la zone de plateau de l'Ader-Doutchi-Maggia d'une altitude moyenne comprise entre 300 et $500 \mathrm{~m}$ et un point culminant à $746 \mathrm{~m}$. Le plan d'eau de la mare est circonscrit entre $5^{\circ} 32^{\prime}$ à $5^{\circ} 51^{\prime}$ de longitude Est et $14^{\circ} 56^{\prime}$ à $15^{\circ} 09^{\prime}$ de latitude Nord (PDC 2010-2014, Commune Rurale de Tabalak) et est formée par trois (3) poches : Silalé, Falé et Kéhéhé (département d'Abalak, 2004). Les apports interannuels sont estimés à 20,6 millions $\mathrm{m}^{3}$ dont 18,2 millions $\mathrm{m}^{3}$ en eaux de ruissellement des koris et 2,4 millions $\mathrm{m}^{3}$ pour les apports pluviométriques directs et c'est un espace sensible aux fluctuations climatiques (ONG YANAHI, 2009).

\section{Matériel}

Les matériels utilisés pour cette étude sont essentiellement composés de :

- fiches d'enquête ;

- appareil numérique de marque NIKON pour les prises de vue ;

- bouteilles en polyéthylène pour le prélèvement des échantillons d'eau ;

- GPS de marque EXPLORIST 200 pour le géo référencement des points ;

- instruments de laboratoire (béchers, éprouvettes graduées, pipette sérologique, poire de sécurité, cuves carrées de 10 ml, plaque chauffante, thermostat) ;

- spectrophotomètre de marque DR 2800

- sonde multi paramètres de marque HANNA pour la mesure in situ de certaines caractéristiques $\quad(\mathrm{pH}, \quad$ température, conductivité électrique, oxygène dissous) de la qualité des eaux ;

- flacons de $500 \mathrm{ml}$ pour le prélèvement des échantillons d'eau ; 


\section{échantillons ;}

- glacière pour la conservation des

- réactifs (hydroxyde de sodium 5,0 N, acide sulfurique $5,25 \mathrm{~N}$, nitriver 3 , nitraver 5 , cyanurate d'ammoniaque, salicylate d'ammoniaque, persulfate de potassium, etc.).

\section{Méthodes}

La méthodologie utilisée dans le cadre de cette étude s'articule autour des principaux axes suivants : la recherche documentaire, les enquêtes, les visites de terrain, les prélèvements et l'analyse des échantillons d'eaux, le traitement et l'analyse des données.

$\mathrm{La}$ recherche documentaire: elle a permis de passer en revue tous les travaux (mémoires, actes de séminaires et colloques, articles de presses et revues, les rapports d'études, les rapports annuels des services) déjà effectuées sur la mare de Tabalak en général et, en particulier sur la variabilité climatique, les zones humides, la gestion des ressources naturelles et le développement local. Les données météorologiques de la station synoptique de Tahoua, située à $60 \mathrm{~km}$ de Tabalak, sur une période de 30 ans (de 1982 à 2012) ont été utilisées pour déterminer la variabilité climatique dans le bassin versant de la mare de Tabalak.

Les enquêtes: elles ont permis de passer la parole à la population afin de cerner sa compréhension sur la variabilité du climat et les impacts sur les productions agricoles autour de la mare. Ce diagnostic a été fait à travers des entretiens collectifs (réunion) et individuels. Au total, trois rencontres avec des groupes mixtes (hommes, femmes et jeunes) ont été organisées et 45 personnes (agriculteurs, éleveurs, pêcheurs, responsables des services de l'Etat, ONGs, projets, société civile, etc.), dont l'âge varie entre 20 et 75 ans ont été interrogées.

Les visites de terrain : trois (3) sorties ont été effectuées afin de recueillir les données géomorphologiques et écologiques à travers des fiches de relevés. Cette activité a été effectuée suivant un transect Nord-Sud afin de parcourir toutes les unités paysagères et comprendre les processus de remplissage de la mare et les problèmes environnementaux qui la menacent (érosion hydrique et éolienne). Cette étape a permis de confirmer ou d'infirmer les informations recueillies lors des entretiens avec la population et les données issues de la recherche bibliographique.

Les prélèvements : douze échantillons d'eau ont été prélevés au niveau de la mare. Ces prélèvements ont été effectués aux mois de février 2013, mai 2013, septembre 2013 et janvier 2014. Ces périodes correspondent aux quatre (4) phases climatiques annuelles de la zone et au régime hydrologique de la mare. Trois (3) points de prélèvements (Tabalak, Pont, Kéhéhé) ont été retenus. Ces points sont situés aux trois (3) sections de la mare en période de basses eaux. Un (1) flacon de $500 \mathrm{ml}$ d'eau est prélevé à chaque échantillonnage.

L'analyse de la qualité des eaux: l'appréciation de la qualité de l'eau de la mare est basée sur un certains nombre de paramètres physico-chimiques, à savoir :

- la température de l'eau qui joue un rôle important en ce qui concerne la solubilité des sels et des gaz ;

- le pH qui renseigne sur l'alcalinité ou l'acidité de l'eau ;

- la conductivité électrique qui détermine le risque salin d'une eau ;

- la teneur en oxygène dissous qui varie de manière journalière et saisonnière ;

- la teneur en ions majeurs (Calcium, Magnésium, Potassium, Sodium);

- les matières en suspension (MES);

- la demande chimique en oxygène (DCO) qui permet de mesurer la teneur en matières organiques totales (excepté quelques composés qui ne sont pas dégradés), y compris celles qui ne sont pas dégradables par les bactéries. Il s'agit donc d'un paramètre important permettant de caractériser la pollution globale d'une eau par des composés organiques ;

- des éléments tels que l'azote $(\mathrm{N})$ et le phosphore $(\mathrm{P})$ qui constituent des éléments nutritifs indispensables aux végétaux ;

- les métaux lourds (densité > à $5 \mathrm{~g} / \mathrm{cm}^{3}$ ), tels que le Cuivre, le Fer, le Manganèse, le Plomb, le Cadmium, le Nickel, le Zinc et le Chrome afin d'apprécier le niveau de pollution de l'eau. 
Les mesures de température, de la conductivité, du $\mathrm{pH}$ et de la teneur en oxygène dissous ont été effectuées, in situ, à l'aide d'une sonde multiparamètrique de marque HANNA. Tandis que les teneurs en ions majeurs, en nitrates, nitrites, phosphore, azote ammoniacal, matières en suspension, demande chimique en oxygène et métaux lourds ont été déterminées par spectrophotométrie dans les Laboratoires des Sciences de la Vie et de la Terre et de Chimie de l'Ecole Normale Supérieure de l'Université Abdou Moumouni de Niamey.

\section{Traitement des données}

Les données météorologiques et les figures ont été réalisées dans Excel. Concernant la dynamique d'occupation des sols, des cartes ont été élaborées avec ArcGis 10 à partir des images satellitaires de 1986 et
2009. Les superficies de toutes les unités d'occupation des sols ont été comparées afin d'analyser les changements intervenus au niveau des différentes unités. Quant aux données d'enquêtes, elles ont été traitées avec le logiciel SPSS 11. Pour déterminer le risque alcalin de l'eau de la mare pour l'irrigation le Ratio d'Absorption de Sodium a été calculé grâce à la formule ci-dessous (Melanie and Jon, 2006) :

$S A R=\frac{N a}{[(C a+M g) / 2]^{1 / 2}}$

Enfin, Les risques potentiels des eaux de la mare pour la consommation humaine et pour la vie aquatique notamment celle des poissons ont été évalués en se référant à certaines normes standards (Ministère de la Santé et des Solidarités, 2005 ; WHO, 2008).

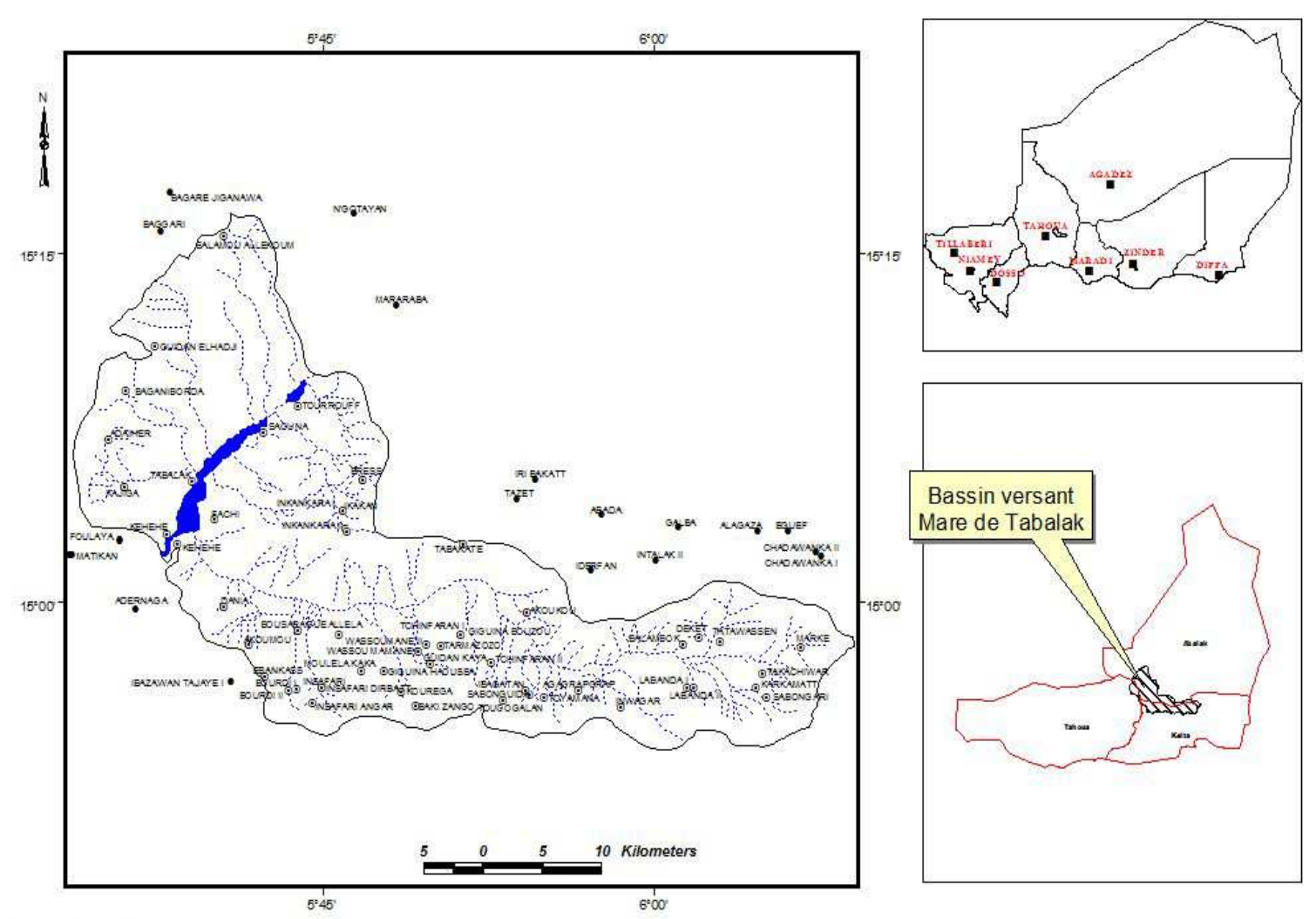

Figure 1 : Carte de localisation du bassin versant de la mare de Tabalak (Nouhou Abdou, 2013). 


\section{RESULTATS}

Variabilité climatique et dynamique des ressources naturelles dans le bassin versant de la mare

La variabilité climatique dans le bassin versant de la mare de Tabalak est surtout marquée par la fluctuation des précipitations, des températures et des vents dans l'année (Figures 2, 3 et 4). Les Figures 5 et 6 montrent la situation comparée des superficies des unités d'occupation des sols entre 1986 et 2009 et fait ressortir les changements intervenus au niveau du bassin versant de la mare de Tabalak.

On remarque sur ces figures, que presque toutes les unités d'occupations des sols ont subi des transformations ou mutations suivant le degré des pressions anthropiques ou naturelles. L'unité koris, a beaucoup plus augmenté par rapport aux autres. Tandis que les unités steppe arbustive et cordon rupicole sont celles qui ont le plus régressées. Ces deux changements s'expliquent d'une part par la déforestation en cours du bassin versant qui accroît la sensibilité du sol à l'érosion hydrique, et d'autre par la conversion des unités vierges à des fins agricoles. Enfin, l'unité dunes vives présente un pourcentage nul.

Perceptions des communautés sur l'impact de la variabilité climatique et des actions anthropiques sur les ressources du bassin versant

Le Tableau 1 présente les perceptions des communautés par rapport aux impacts de la variabilité climatique et des actions anthropiques sur les ressources et les activités agricoles.

Ces impacts se traduisent sur le plan :

- agricole, par la perturbation des saisons et la baisse de la production ;

- pastorale, par la dégradation des ressources à valeur pastorale au profit des espèces non appétées par les animaux et la persistance des épizooties ;

- halieutique, par la baisse des captures, la baisse du régime hydrologique de la mare, l'apparition des plantes aquatiques proliférantes comme le Typha australis.

\section{Etat de la qualité des eaux de la mare}

Les résultats des analyses sur les paramètres physico-chimiques des eaux de la mare en fonction du lieu et de la date de prélèvement sont représentés dans le Tableau 2.

La température est plus élevée en mai au niveau de Tabalak $\left(30,76{ }^{\circ} \mathrm{C}\right)$ et en septembre au niveau de Kéhéhé $\left(31,66{ }^{\circ} \mathrm{C}\right)$. Par contre, la conductivité est plus élevée au niveau du pont $(380 \mu \mathrm{S} / \mathrm{cm}$ au mois de janvier et $338 \mu \mathrm{S} / \mathrm{cm}$ en février). Le $\mathrm{pH}$ est plus élevé en Janvier (entre 8 et 8,32). Tandis que, le taux en oxygène dissous est plus élevé en Janvier au niveau de Kéhéhé $(10,17 \mathrm{mg} / \mathrm{l})$ et en février au niveau de Tabalak $(8,22 \mathrm{mg} / \mathrm{l})$. Les taux en nitrates sont plus élevés en janvier au niveau de Kéhéhé $(20 \mathrm{mg} / \mathrm{l})$ et de Tabalak $(19 \mathrm{mg} / \mathrm{l})$. Il varie faiblement en fonction du lieu de prélèvement pour les autres mois. Cela est aussi valable pour le taux en nitrites. Les taux en phosphore et en azote ammoniacal sont de l'ordre de 1,6 et $1,5 \mathrm{mg} / \mathrm{l}$ au niveau de Kéhéhé et de $1,6 \mathrm{mg} / \mathrm{l}$ et $1,1 \mathrm{mg} / \mathrm{l}$ au niveau de Tabalak. La demande chimique en oxygéné (DCO) et les matières en suspension (MES) sont plus élevées en mai et janvier. En janvier, la DCO est de l'ordre de 157 et $153 \mathrm{mg} / \mathrm{l}$ à Kéhéhé et Tabalak et les MES 416 et $268 \mathrm{mg} / \mathrm{l}$ à Kéhéhé et Tabalak. Les taux en $\mathrm{Na}^{+}$et $\mathrm{Ca}^{2+}$ sont élevés en janvier surtout au niveau de Kéhéhé où ils sont respectivement de 2,8 et $4,2 \mathrm{mg} / \mathrm{l}$. La concentration en $\mathrm{Mg}^{2+}$ est moins élevée en septembre au niveau de Kéhéhé $(2,15 \mathrm{mg} / \mathrm{l})$. Tandis que celle en $\mathrm{K}^{+}$est plus élevée surtout en janvier au niveau de Kéhéhé $(4,1 \mathrm{mg} / \mathrm{l})$. Quant au Fer, il est plus élevée à Tabalak (1,266 mg/l) et Kéhéhé $(1,292 \mathrm{mg} / \mathrm{l})$, alors que le Manganèse est présent uniquement à Tabalak avec une teneur de l'ordre $0,036 \mathrm{mg} / \mathrm{l}$. Le plomb $(0,233 \mathrm{mg} / \mathrm{l})$, le chrome $(0,124 \mathrm{mg} / \mathrm{l})$ et le zinc $(0,24 \mathrm{mg} / \mathrm{l})$ sont plus élevées à Tabalak; alors que le nickel est plus élevé à Kéhéhé avec 0,133 $\mathrm{mg} / \mathrm{l}$. 


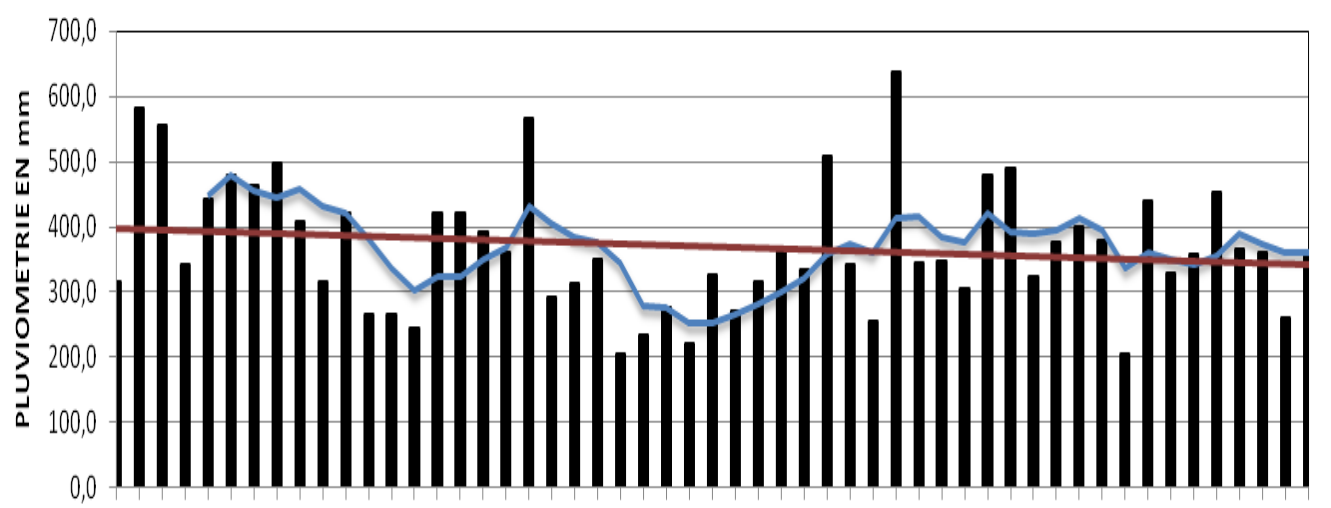

196019621964196619681970197219741976197819801982198419861988199019921994199619982000200220042006200820102012

ANNEES

DLUIESEN mm

Figure 2 : Evolution de la pluviométrie annuelle à Tahoua de 1960 à 2012.

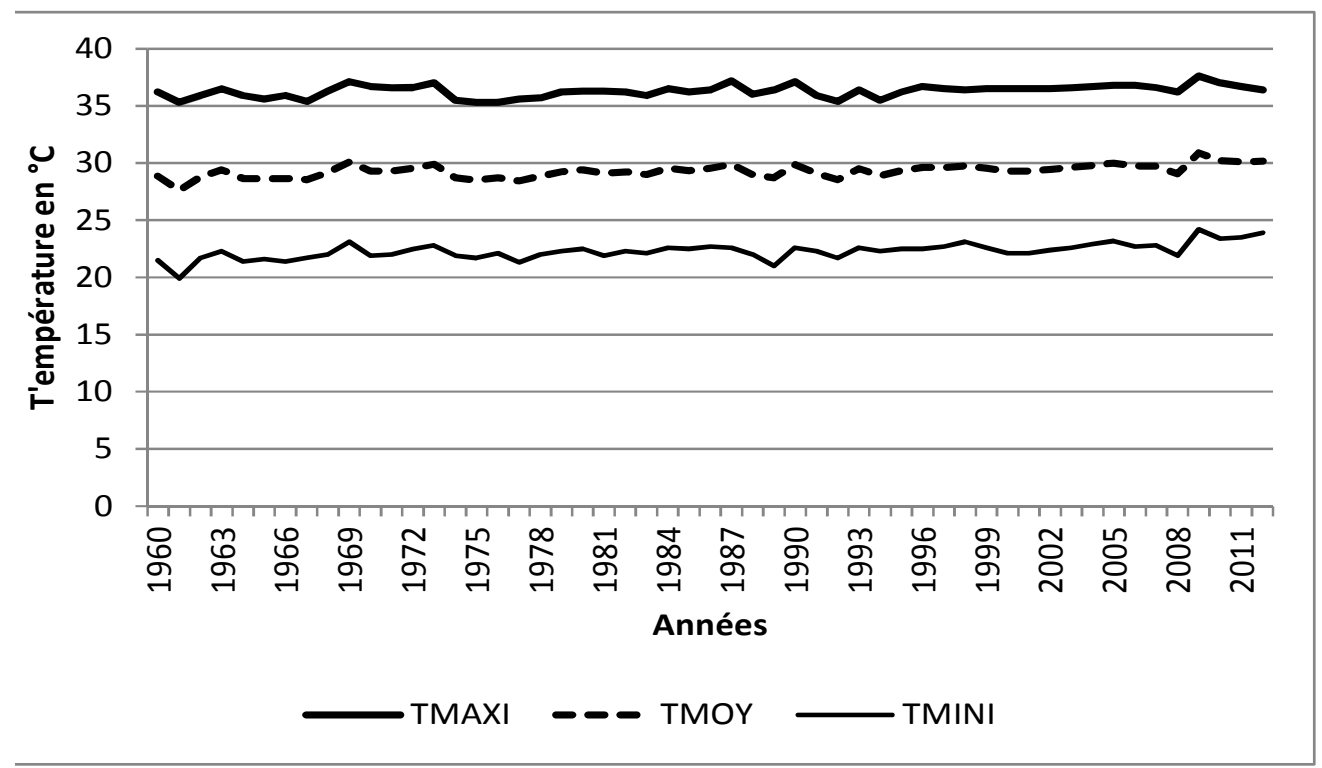

Figure 3: Evolution des températures maximales, minimales et moyennes à Tahoua de 1960 à 2012. 


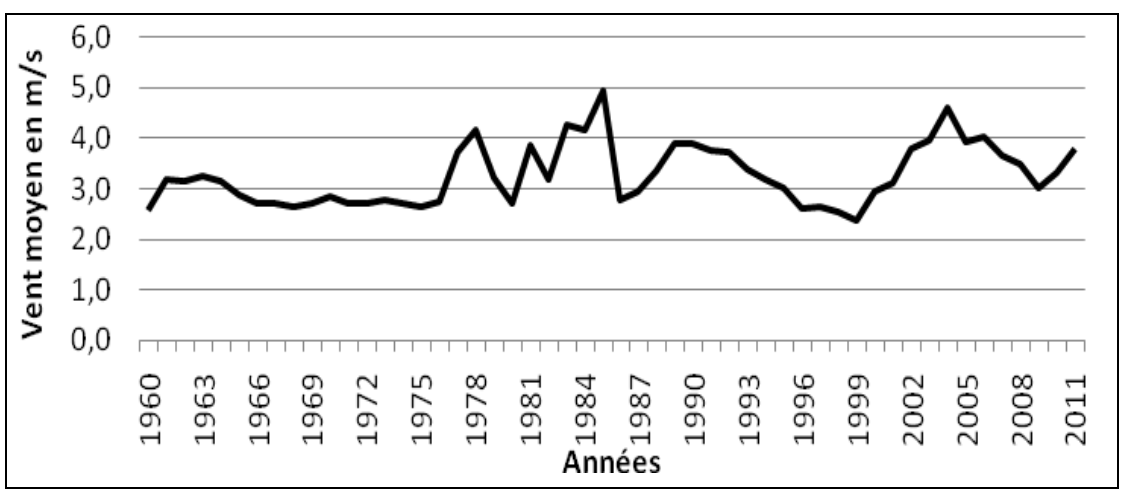

Figure 4 : Evolution de la vitesse moyenne du vent de 1960 à 2012.

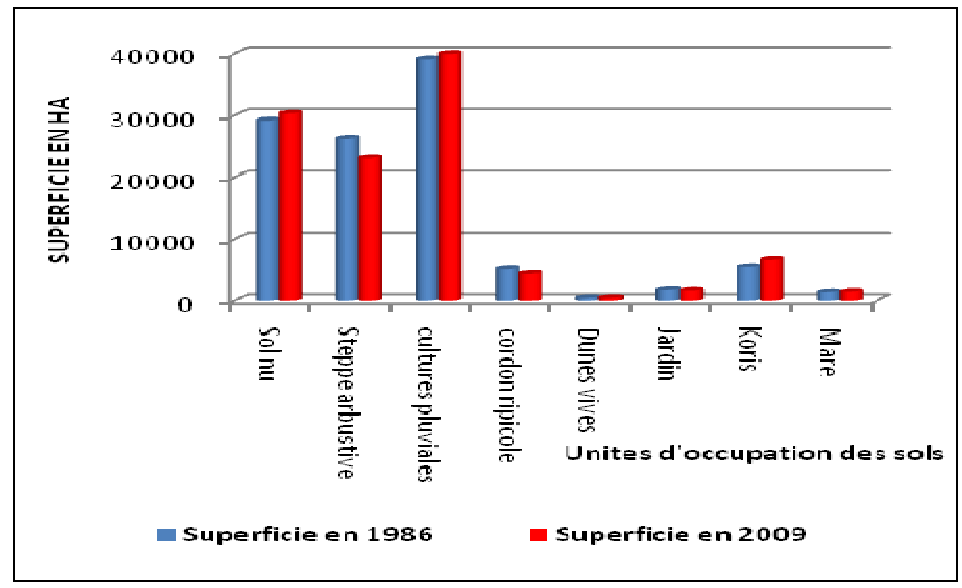

Figure 5 : Superficies comparées des unités paysagères entre 1986 et 2009.

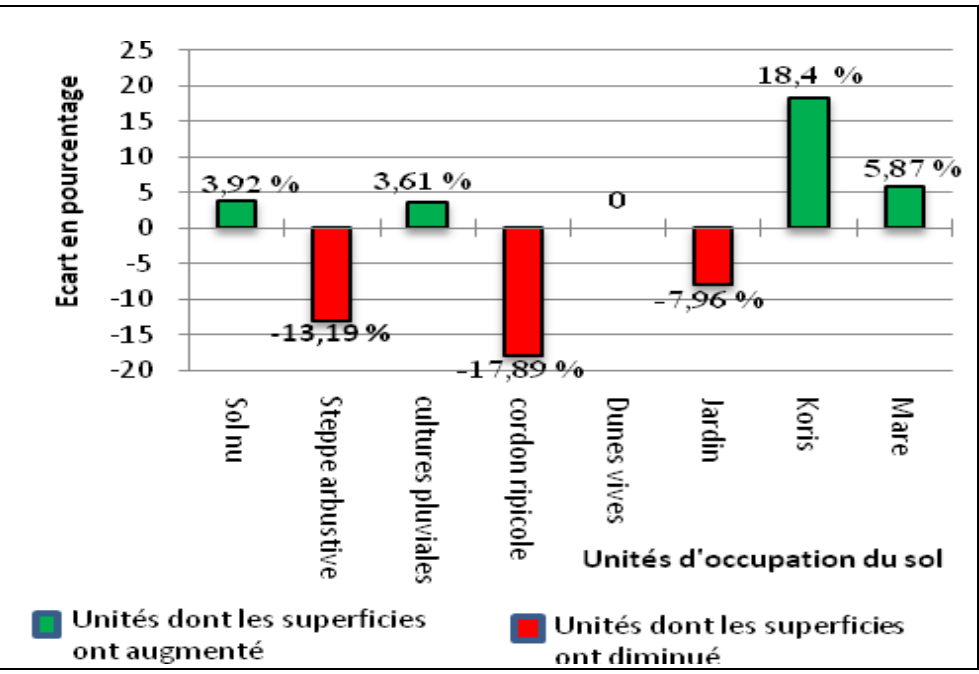

Figure 6 : Ecarts des différentes unités paysagères entre 1986 et 2009. 
Tableau 1 : Perceptions des communautés sur les impacts du climat.

\begin{tabular}{|c|c|c|}
\hline Activités & Perceptions des impacts & Stratégies locales d'adaptation \\
\hline Agriculture & $\begin{array}{l}\text { - perturbation des saisons : démarrage tardif de la saison des pluies et arrêt } \\
\text { précoce des précipitations ; } \\
\text { - baisse des productions des cultures pluviales ; } \\
\text { - perte des terres par ravinement et formation de dunes vives ; } \\
\text { - forte pression des ennemis de culture ; } \\
\text { - baisse du volume d'eau de la mare et de la nappe phréatique }\end{array}$ & $\begin{array}{l}\text { - modification du calendrier cultural; } \\
\text { - diversification des cultures ; } \\
\text { - diversification des sources de revenus; } \\
\text { - adoption de nouvelles techniques d'irrigation économes } \\
\text { d'eau; } \\
\text { - actions de protection de l'environnement (comme la } \\
\text { régénération naturelle assistée, apport de fumure, paillage). }\end{array}$ \\
\hline Elevage & $\begin{array}{l}\text { - amenuisement progressif des parcours et une dégradation continue des } \\
\text { ressources pastorales ; } \\
\text { - diminution des espèces appétées au profit des espèces sans valeur } \\
\text { pastorales ; } \\
\text { - persistance des épizooties. }\end{array}$ & $\begin{array}{l}\text { - utilisation de compléments alimentaires pour les } \\
\text { animaux ; } \\
\text { - réduction de la taille du troupeau pour les grands éleveurs } \\
\text { afin de faire face à la rareté de pâturages ; } \\
\text { - diversification des sources de revenus (petit commerce). }\end{array}$ \\
\hline Pêche & $\begin{array}{l}\text { - baisse des captures; } \\
\text { - ensablement de la mare; } \\
\text { - assèchement fréquent de la mare ; } \\
\text { - disparition de certaines espèces de poissons ; } \\
\text { - baisse de la qualité des eaux (turbidité, couleur,...); } \\
\text { - prolifération de plantes aquatiques envahissantes (comme Typha } \\
\text { australis). }\end{array}$ & $\begin{array}{l}\text { - fermeture de la mare pour les activités de pêche; } \\
\text { - empoissonnement ; } \\
\text { - captures sélectives par utilisation d'engins de pêche } \\
\text { appropriés ; } \\
\text { - construction de puisards pour conserver les géniteurs en } \\
\text { cas d'assèchement de la mare ; } \\
\text { - faucardage des plantes envahissantes. }\end{array}$ \\
\hline
\end{tabular}


M. M. ADAMOU et al. / Int. J. Biol. Chem. Sci. 9(3): 1665-1677, 2015

Tableau 2 : Résultats des analyses physico-chimiques de la mare.

\begin{tabular}{|c|c|c|c|c|c|c|c|c|c|c|c|}
\hline & \multicolumn{3}{|c|}{ Mars 2013} & \multicolumn{3}{|c|}{ Mai 2013} & \multicolumn{3}{|c|}{ Septembre 2013} & \multicolumn{2}{|c|}{ Janvier 2014} \\
\hline & Pont & Tabalak & Kéhéhé & Pont & Tabalak & Kéhéhé & Pont & Tabalak & Kéhéhé & Pont & Tabalak \\
\hline Température $\left(\mathrm{en}^{\circ} \mathrm{C}\right)$ & 26,17 & 27,09 & 26,56 & 30,17 & 30,76 & 29,64 & 31,05 & 30,38 & 31,66 & 19,28 & 20,24 \\
\hline Conductivité (en $\mu \mathrm{S} / \mathrm{cm})$ & 338 & 289 & 291 & 263 & 244 & 239 & 275 & 196 & 240 & 380 & 343 \\
\hline DO $\%$ & 84,7 & 108,2 & 102,5 & - & - & - & - & - & - & 43,6 & 42,7 \\
\hline Oxygène dissous (mg/l) & 6,54 & 8,22 & 7,81 & - & - & - & - & - & - & 3,82 & 3,68 \\
\hline $\mathrm{pH}$ & 6,12 & 6,51 & 6,92 & 6,31 & 6,18 & 6,41 & 6,26 & 6,5 & 6,82 & 8 & 8,32 \\
\hline Nitrate (en mg/l) & 0,3 & 0,3 & 0,3 & 0,3 & 2 & 4 & 0,1 & 0,3 & 0,1 & 2 & 2 \\
\hline Nitrite (en $\mathrm{mg} / \mathrm{l}$ ) & 0,05 & 0,04 & 0,02 & 0,04 & 0,02 & 0,06 & 0,03 & 0,012 & 0,005 & 0,09 & 0,09 \\
\hline Phosphate total (en mg/l) & 0,33 & 0,2 & 0,4 & 0,11 & 0,73 & 0,15 & 0,28 & 0,73 & 0,45 & 1 & 1,6 \\
\hline Azote ammoniacal (en mg/l) & 0,29 & 0,12 & 0,11 & 0,27 & 0,33 & 0,28 & 0,1 & 0,1 & 0,28 & 0,4 & 1,1 \\
\hline $\mathrm{DCO}$ (en mg/l) & 17,8 & 33,1 & 30,3 & 79,4 & 86,9 & 98 & 11,1 & 7,37 & 14,8 & 81,5 & 81 \\
\hline MES (en mg/l) & 26 & 57 & 54 & 144 & 250 & 195 & 62 & 103 & 190 & 200 & 268 \\
\hline $\mathrm{Ca}($ en $\mathrm{mg} / \mathrm{l})$ & 0,495 & 0,435 & 0,4 & 0,29 & 0,25 & 0,21 & 0,36 & 0,35 & 0,38 & 1,7 & 1,6 \\
\hline $\mathrm{Mg}$ (en mg/l) & 5,66 & 5,46 & 5 & 3,3 & 5 & 6 & 2,77 & 2,83 & 2,15 & 4,4 & 4,08 \\
\hline $\mathrm{Na}($ en $\mathrm{mg} / \mathrm{l})$ & 0,26 & 0,262 & 0,259 & 1,27 & 1,26 & 1,15 & 18,4 & 16,5 & 16,3 & 1,1 & 1,2 \\
\hline $\mathrm{Fe}($ en $\mathrm{mg} / \mathrm{l})$ & 0,339 & 1,266 & 1,292 & - & - & - & - & - & - & - & - \\
\hline Mn (en mg/l) & abs & 0,036 & abs & - & - & - & - & - & - & - & - \\
\hline $\mathrm{Pb}($ en $\mathrm{mg} / \mathrm{l})$ & 0,163 & 0,233 & 0,199 & - & - & - & - & - & - & - & - \\
\hline $\mathrm{Ni}$ (en $\mathrm{mg} / \mathrm{l}$ ) & 0,122 & 0,089 & 0,133 & - & - & - & - & -- & & -- & - \\
\hline $\mathrm{Zn}(\mathrm{en} \mathrm{mg} / \mathrm{l})$ & 0,075 & 0,24 & 0,211 & - & - & - & - & - & - & - & - \\
\hline $\mathrm{Cr}($ en $\mathrm{mg} / \mathrm{l})$ & 0,111 & 0,124 & 0,045 & - & - & - & - & - & - & - & - \\
\hline
\end{tabular}




\section{DISCUSSION}

Variabilité climatique et dynamique des ressources naturelles dans le bassin versant de la mare

Cette étude montre bien que la dynamique de l'état des ressources rime bien avec l'évolution du climat et de l'action de l'homme dans le bassin versant de la mare. L'étude diachronique des images de 1986 et 2009, montre de manière claire l'évolution de l'occupation des sols entre les deux années. D'une manière générale, cette étude montre que l'environnement biophysique du bassin versant de la mare de Tabalak présente de sérieuses tendances à la dégradation des terres. Ainsi, à l'échelle des paysages, on assiste à la fragmentation des formations végétales due à la mise en culture, la réduction de la superficie des zones de parcours, la formation de dunes vives surplombant la mare. Et, à l'échelle de l'occupation des sols ; c'est la dominance des zones cultivées au détriment des zones de parcours; ce qui entraîne une augmentation de la charge animale, le développement de l'agriculture pluviale extensive sur des terres de plus en plus marginales, le développement des cultures irrigués avec comme conséquence, une altération de la qualité de l'eau.

Ainsi, la tendance à la dégradation des terres dont les conséquences directes affectent l'eau de la mare tant en quantité qu'en qualité est particulièrement due aux facteurs climatiques (baisse des pluviométries, hausse des températures, etc.) et à la surexploitation des ressources. Aussi, le processus d'ensablement de la mare qui se trouve entourée par un cordon de dunes de sable dans la partie Nord et Nord-Est et des koris dans la partie Est et Sud/Est affecte sérieusement le niveau de remplissage de la mare. Les conséquences sont d'une part, l'apparition du phénomène de ruissellement diffus sur les plateaux et les pentes, entraînant la formation et l'élargissement des koris et des ravins qui sont très perceptibles dans la zone. Et d'autre part, l'érosion éolienne créant les conditions propices pour la formation de vastes glacis et des dunes vives.
Ces impacts perçus et cités par les populations au cours de l'enquête montrent bien que la variabilité climatique et l'action de l'homme ont engendré des modifications sur l'état des ressources et sur les bases productives au niveau du bassin versant de la mare de Tabalak.

Les actions de l'homme conjuguées surtout aux facteurs climatiques demeurent les causes fondamentales de toutes les transformations survenues. Au cours de ces trente dernières années, un enchaînement d'événements climatiques d'une ampleur et d'une rapidité sans précédent, ont sérieusement entamée l'état des ressources dans le bassin versant de la mare. On peut penser notamment aux périodes de sécheresse qui ont grandement affectés les écosystèmes ainsi que les systèmes de production. Cela confirme les résultats des études menées par le LUCOP-TAN (2004, 2009) sur l'état de la mare de Tabalak.

Certains auteurs comme Maton et al. (2010) et des organismes tel que l'Institut International de Recherche sur les Politiques Alimentaires (2009), ont établi une étroite relation entre la variabilité climatique et les productions agropastorales (agriculture, élevage et pêche), d'une part, et entre la variation des précipitations et de la température sur le régime et la qualité des eaux, d'autre part.

\section{Etat de la qualité des eaux de la mare}

En ce qui concerne la qualité des eaux de la mare, les mesures de la conductivité indiquent une eau douce avec une minéralisation faible à moyen. En effet, la conductivité électrique mesurée est de 198 à $380 \mu \mathrm{S} / \mathrm{cm}$. Cette dernière est en apparente augmentation selon l'étude menée par Studi en 2001. En se basant sur le diagramme de Riverside, les conductivités enregistrées montrent que le risque salin pour l'utilisation de l'eau de cette mare en irrigation est faible à moyen selon les saisons. Les ordres de grandeurs des paramètres chimiques des eaux de la mare montrent que la qualité physicochimique est relativement bonne. 
Le $\mathrm{pH}$ de 6,12 à 8,32 montre que les eaux de la mare sont proches de la neutralité. Ces valeurs ne sont pas significativement différentes de celles trouvées par Studi en 2001 ( $\mathrm{pH}=9$ ) au niveau de la mare de Tabalak $(\mathrm{P}>0,05)$.

La température mesurée est fonction des saisons à tous les points de mesure. Ces valeurs sont proches de celles trouvées par Studi en 2001 au niveau de Tabalak et montrent qu'il n'existe pas de risque en ce qui concerne la solubilité des sels et des gaz dont, entre autres, l'oxygène nécessaire à l'équilibre de la vie aquatique. Par ailleurs, elles ne montrent pas un risque élevé d'accélération des vitesses des réactions chimiques et biochimiques.

Le taux en oxygène dissous de la mare indique que l'eau est de bonne qualité pour la vie aquatique même si le niveau de l'eau baisse considérablement au cours de l'année. Néanmoins, les valeurs enregistrées au niveau du Pont et Tabalak sont assez faibles en janvier 2014 avec la respiration nettement supérieure à la production. En mars 2013 et janvier 2014, cette dernière est supérieure à la respiration respectivement à Tabalak et Kéhéhé. Les concentrations en oxygène de la mare varient en fonction des saisons et cela confirme les écrits de Richoux et Lebreton (2008) qui soutiennent que la hausse des températures associée à une baisse des précipitations entraînerait une baisse du niveau des plans d'eaux peu profonds et de la concentration en oxygène dissous comme c'est le cas pour la mare de Tabalak.

Les concentrations en nitrates et nitrites des eaux de la mare montrent qu'elles ne sont pas globalement polluées par le lessivage des terres après épandage d'engrais, ni par les eaux usées domestiques ou certaines eaux usées à caractère basique qui constituent les sources directes de ces ions pour les eaux de surface.

Celles en phosphore total et azote ammoniacal sont largement élevées en janvier, période de décrue de la mare et aussi du fait que la mare s'est asséchée au niveau de Kéhéhé à cette période. En plus, les échantillons prélevés sont constitués des eaux drainées pour l'irrigation. Ces valeurs indiquent une altération de la qualité de l'eau de la mare par le phosphore et l'ammonium en janvier 2014 au niveau des trois points de prélèvement (Pont, Tabalak et Kéhéhé). L'altération par le phosphore est également observée en septembre 2013 au niveau de Tabalak et Kéhéhé, en mai 2013 à Tabalak puis en mars 2013 au Pont et à Kéhéhé. Celle en ammonium est également constatée à Tabalak en mai 2013.

Les concentrations des matières en suspension (MES) varient en fonction de la saison. Les valeurs obtenues aux trois points de prélèvement sont plus élevées en janvier car à cette période le niveau de l'eau a considérablement baissé et aussi du fait du passage des animaux en transhumance autour de la mare. Elles ne sont pas de nature à affecter significativement la transparence de l'eau et diminuer la pénétration de la lumière ni gêner la respiration des poissons ou accumuler des quantités élevées de matières toxiques (métaux, pesticides, huiles minérales, hydrocarbures aromatiques polycycliques, etc.).

En mai et janvier, les trois points de prélèvement ont des concentrations en DCO les plus élevées indiquant ainsi une altération de la qualité des eaux par la matière organique.

Le Ratio Absorption de Sodium montre également que le risque alcalin des eaux pour l'irrigation est faible surtout si la mare est remplie d'eau comme c'est le cas en février. Et, ces valeurs sont proches de celles obtenues en 2001 par Studi (SAR entre 0,13 et 0,18 ) au niveau de la mare de Tabalak.

La présence des métaux lourds tels que le fer, le zinc, le nickel, le plomb, le manganèse, et le chrome montre l'existence des sources de pollutions diffuses surtout à Tabalak où les teneurs sont plus élevées. Il s'agit des rejets provenant des apports, dus aux transports routiers surtout pour le plomb et des eaux de ruissellement, accentués par les érosions et des matériaux galvanisés (toitures métalliques, gouttières) surtout pour le zinc. 
Les faibles teneurs mesurées indiquent que les eaux de la mare ne sont pas polluées. Néanmoins, le suivi des concentrations des éléments présents est particulièrement important vu leur capacité de bioaccumulation le long du réseau trophique.

Enfin, les valeurs obtenues lors des différentes analyses sur la qualité de l'eau montrent que même si certains paramètres restent dans les normes requises, le suivi des concentrations de certains éléments comme les métaux lourds et les teneurs en phosphore, en demande chimique en oxygène et en azote ammoniacal doit être envisagé.

\section{Conclusion}

$\mathrm{Au}$ terme de cette étude, le constat général montre que l'environnement biophysique du bassin versant de la mare de Tabalak présente de sérieuses tendances à la dégradation des terres. Les conséquences directes de ces tendances sont l'affectation du potentiel productif du bassin versant de la mare particulièrement par les facteurs climatiques et la surexploitation des ressources. En ce qui concerne la qualité de l'eau de la mare, même si elle est écologiquement acceptable au vu des résultats des analyses, les concentrations de certains paramètres indiquent une certaine pollution. Le suivi des paramètres physico-chimiques doit être régulier et s'impose comme un prérequis indispensable pour faire face aux éventuels impacts de la variabilité climatique.

\section{REMERCIEMENTS}

Les auteurs remercient le Programme d'Appui aux Centres d'Excellence Régionaux de l'Union Monétaire Ouest-Africaine (PACER-UEMOA) pour l'appui financier apporté dans la réalisation de cette étude.

\section{REFERENCES}

Alhouza M. 2010. Diagnostic des problèmes de gestion de la mare de Tabalak, zone humide d'importance internationale de la convention RAMSAR: Proposition d'un mode de gestion durable. Mémoire de DESS, IUB de Cotonou, p. 113.
Bolawa OE, Gbenle GO. 2005. Analysis of industrial impacts on physiochemical parameters and heavy metal concentrations in water of river Majidum, Molatori and Ibeshe around Ikorodu in Lagos, Nigeria. Journal of Environmental Science and Water Ressources, 1(2): 34-38.

Chavan BC. 2012. Sewage treatment with constructed Westland using Panicum maxima forage grass. Journal of Environmental Science and Water Ressources, 1(9): 223-230.

Conseil National de l'Environnement pour un Développement Durable. 2011. Impacts des changements climatiques dans le secteur des ressources en eau. Rapport final, Niamey, Niger, p. 58.

Direction départementale de l'environnement de Malbaza. 2013. Résultats d'analyse de la qualité de l'eau de la mare de Guidan Magagi. Rapport, p. 15.

Institut International de Recherche sur les Politiques Alimentaires. 2009. Changements Climatiques : Impacts sur l'agriculture et coûts de l'adaptation. Rapport final, Washington DC, USA, p. 30.

Laboratoire Interuniversitaire des Sciences de l'Education et de la Communication. 2004. Qualité physico-chimique des eaux de surface. Rapport, IBGE. Mis en ligne le 25 janvier 2010, consulté le 10 juin 2013. fr.wikipédia.org./wiki/Portail : eau.

Maton L, Rinaudo JD, Chazot S. 2010. Demande en eau agricole: Impact des changements climatiques et adaptations. Réunion de clôture du Projet VULCAIN (9 Novembre 2010, Collioure).

Melanie LC, Jon PM, 2006. Water-Quality Characteristics, Including SodiumAdsorption Ratios, for Four Sites in the Powder River Drainage Basin, Wyoming and Montana, Water Years 2001-2004. U.S. Geological Survey: Reston, Virginia ; 32 p.

Ministère de l'Hydraulique, de l'Environnement et de la Lutte Contre la 
Désertification du Niger. 2006. Diagnostic sur la gestion des zones humides du Niger. Rapport final, p. 35.

Ministère de la Santé et des Solidarités, 2005. La qualité de l'eau potable en France Aspects sanitaires et réglementaires. Dossier d'information. République Française, 43 p.

ONG YANAYI, 2009. Appui à la mise en place des mécanismes de gestion durable et concertée de la mare de Tabalak. Document de Projet, 14 p.

Onunkwo A, UJeoije AP, Igbokwe B, Ikoroji IL. 2012. Underground water and pollution vulnerability assessment of lower Imo sedimentary basin of southestearn Nigeria. Journal of Environmental Science and Water Ressources, 1(4): 105-114.

Programme des Nations Unies pour le développement (PNUD), Bureau de la prévention des crises et du relèvement (BCPR). 2013. Gestion Durable des zones Humides Face aux Risques
Climatiques au Niger : Le Cas de la Mare de Tabalak. UNDP BCPR: New York, NY ; 57.

Projet de Lutte Contre la Pauvreté/Antenne Tahoua Nord. 2009. Etude sur la mise en place des mécanismes de gestion concertée et durable autour des ressources naturelles de la mare de Tabalak. Rapport, p. 89.

RAMSAR. 2013. http:// www.ramsar .org. Consulté le 24 Avril 2013.

Richoux P, Lebreton P. 2008. L'Evolution Climatique et son Impact en Dombes. Les cahiers de la fondation Pierre Vérots 1 : Paris, France ; 20.

Studi. 2001. Projet de développement hydroagricole de Kéhéhé. Etude de Faisabilité. Rapport, Hydrologie-hydrogéologie, p. 52.

World Health Organization. 2008. Guideline for Drinking-Water Quality, Incorporating the First and Second Addenda. (vol 1, $3^{\text {rd }}$ edn). World Health Organization : Geneva ; 668. 